\title{
Notch signaling contributes to the maintenance of both normal neural stem cells and patient-derived glioma stem cells
}

Yi-Yang Hu ${ }^{1 \dagger}$, Min-Hua Zheng ${ }^{1 \dagger}$, Gang Cheng ${ }^{2}$, Liang Li ${ }^{2}$, Liang Liang ${ }^{1}$, Fang Gao ${ }^{1}$, Ya-Ning Wei ${ }^{1}$, Luo-An Fu² Hua $\operatorname{Han}^{1 *}$

\begin{abstract}
Background: Cancer stem cells (CSCS) play an important role in the development and recurrence of malignant tumors including glioma. Notch signaling, an evolutionarily conserved pathway mediating direct cell-cell interaction, has been shown to regulate neural stem cells (NSCs) and glioma stem cells (GSCs) in normal neurogenesis and pathological carcinogenesis, respectively. However, how Notch signaling regulates the proliferation and differentiation of GSCs has not been well elucidated.

Methods: We isolated and cultivate human GSCs from glioma patient specimens. Then on parallel comparison with NSCs, we inhibited Notch signaling using $\gamma$-secretase inhibitors (GSI) and assessed the potential functions of Notch signaling in human GSCs.

Results: Similar to the GSI-treated NSCs, the number of the primary and secondary tumor spheres from GSI-treated GSCs decreased significantly, suggesting that the proliferation and self-renewal ability of GSI-treated GSCs were attenuated. GSI-treated GSCs showed increased differentiation into mature neural cell types in differentiation medium, similar to GSI-treated NSCs. Next, we found that GSI-treated tumor spheres were composed of more intermediate progenitors instead of CSCS, compared with the controls. Interestingly, although inhibition of Notch signaling decreased the ratio of proliferating NSCs in long term culture, we found that the ratio of G2+M phase-GSCs were almost undisturbed on GSI treatment within $72 \mathrm{~h}$.

Conclusions: These data indicate that like NSCs, Notch signaling maintains the patient-derived GSCs by promoting their self-renewal and inhibiting their differentiation, and support that Notch signal inhibitor GSI might be a prosperous candidate of the treatment targeting CSCs for gliomas, however, with GSI-resistance at the early stage of GSCs cell cycle.
\end{abstract}

\section{Background}

Glioma, the most common tumor of the central nervous system (CNS), frequently leads to death. Glioma is derived from brain glial tissue and comprises several diverse tumor forms and grades. Treatment of malignant gliomas is often palliative due to their infiltrating

\footnotetext{
* Correspondence: fuluoan@fmmu.edu.cn; huahan@fmmu.edu.cn + Contributed equally

'State Key Laboratory of Cancer Biology, Department of Medical Genetics and Developmental Biology, Fourth Military Medical University, Chang-Le Xi Street \#17, Xi'an 710032, PR China

${ }^{2}$ Department of Neurosurgery, Xijing Hospital, Fourth Military Medical University, Chang-Le Xi Street \#17, Xi'an 710032, PR China

Full list of author information is available at the end of the article
}

nature and high recurrence. Despite advances in surgery, chemotherapy and radiation gradually result in therapyresistance. However, genetic events that lead to gliomas are mostly unknown.

Recent researches highlight the importance of cancerinitiating cells in the malignancy of gliomas [1-3]. These cells have been referred to as glioma stem cells (GSC), as they share similarities to normal neural stem cells (NSCs) in the brain. There is increasing evidence that malignant gliomas arise from and contain these minority tumor cells with stem cell-like properties. This subpopulation of tumor cells with the potential for self-renewal and multi-lineage differentiation that recapitulates the

\section{Biomed Central}

(c) 2011 Hu et al; licensee BioMed Central Ltd. This is an Open Access article distributed under the terms of the Creative Commons Attribution License (http://creativecommons.org/licenses/by/2.0), which permits unrestricted use, distribution, and reproduction in any medium, provided the original work is properly cited. 
phenotype of the original glioma [4-8], plays an important role in glioma initiation, growth, and recurrence. Eliminating GSCs from the bulk tumor mass seems to be a prosperous therapeutic strategy $[9,10]$. Therefore, it is extremely important to understand the signal pathways that contribute to the formation and maintenance of GSCs.

A number of signal pathways are involved in the formation and maintenance of stem cells, many of which are closely conserved across species. Notch signaling, an evolutionarily conserved pathway mediating direct cellcell interaction and signaling, plays a pivotal role in the maintenance of NSCs [11]. The functions of the Notch pathway in cancer formation have been gradually established, and recent data have also implicated a role for Notch signaling in GSCs [12]. Notch is a family of hetero-dimeric transmembrane receptors composed of an extracellular domain responsible for ligand recognition, a transmembrane domain, and an intracellular domain involved in transcriptional regulation. When Notch receptor is triggered by the ligands on the neighboring cells, the intracellular domain of the Notch receptor (NICD) is released from the membrane, after successive proteolytic cleavages by the $\gamma$-secretase complex $[13,14]$. NICD then translocates into the nucleus and associates with the transcription factor RBP-J, the DNA recombination signal binding protein-J $\kappa$. The NICD-RBP-J complex further recruits other co-activators, and activates the expression of downstream genes associated with cell proliferation, differentiation and apoptosis [15]. It is believed that $\gamma$-secretase inhibitors (GSI) decrease the activity of Notch signaling and slow the growth of Notch-dependent tumors such as medulloblastoma [12].

Rapid proliferation, self-renewal ability and multipotential differentiation are the hallmarks of both normal NSCs and GSCs. Similarities in the growth characteristics and gene expression patterns of normal NSCs and brain tumor CSCs suggest that pathways important for NSCs are probable targets for eliminating brain tumor CSCs. The RBP-J-mediated canonical Notch pathway plays several significant roles in the maintenance and differentiation of NSCs [16-18]. During embryogenesis, Notch signaling is required to maintain all NSC populations, and to repress the differentiation of NSCs into intermediate neural progenitors (INPs) in vivo [19-21]. Along with later development, Notch signal commits NSCs to an astroglia fate, while repressing neuronal differentiation [22]. In adult, Notch signaling modulates cell cycle in order to ensure brain-derived NSCs retain their self-renewal property [23].

Increasing evidence has shown that there is a link between tumorigenesis and aberrantly activated Notch signaling [24,25]. Notch1 and its ligands, Dll1 and Jagged1, were overexpressed in many glioma cell lines and primary human gliomas. When the expression of Notch1, Dll1 or Jagged1 was down-regulated by RNA interference, apoptosis and proliferation inhibition in multiple glioma cell lines were induced [26]. Depletion of Hey1, a member of Hes-related family downstream effectors of Notch signaling, by RNA interference also reduces proliferation of glioblastoma cells in tissue culture [27]. Moreover, the blockade of Notch signaling directly caused cell cycle exit, apoptosis, differentiation, and reduced the CD133-positive cells in medulloblastoma and glioblastoma cell lines while Notch activation enhances the expression of Nestin, promotes cell proliferation and the formation of NSC-like colonies and plays a contributing role in the brain tumor stem cells [28-30]. However, the exact roles of Notch signaling in the proliferation and differentiation of patient-derived GSCs have not been clearly elucidated.

In this study, we explore the roles of Notch signaling in patient-derived GSCs with parallel analysis of normal NSCs by using GSI-mediated inhibition of Notch signaling in vitro. The results showed that when Notch signaling was inhibited, the proliferation and self-renewal ability of GSCs from human primary gliomas were attenuated. In addition, the blockade of Notch signaling in GSCs increased their differentiation into the downstream neural cell types, and promoted their conversion from stem cells into INP-like cells. Interestingly, although inhibition of Notch signaling definitely decreased the proliferating GSCs in long term culture, we found that the percentage of $\mathrm{G} 2+\mathrm{M}$ phase-GSCs were almost undisturbed at the initial stage of GSI treatment. To summarize, our results suggested that Notch signaling maintained GSCs by promoting their selfrenewal and inhibiting their differentiation into INP-like cells, and supported that Notch signal inhibitors might be prosperous candidates of the treatments targeting CSCs for gliomas.

\section{Methods}

\section{Glioma samples}

Glioma tissues were obtained from 9 adult patients with pathologically diagnosed grade 2 to grade 4 gliomas, at the Department of Neurosurgery in Xijing Hospital, Fourth Military Medical University, under the guidance from the Medical Ethnic Committee of the Fourth Military Medical University. The summary of the patient population is outlined in Additional file 1: Table S1.

\section{Neurosphere culture}

Neurosphere cultures were performed as described previously with some modifications [21]. Briefly, for the culture of NSCs, the brains from embryonic (E) day 12.5 C57BL/6 mice were dissected under a stereomicroscope. And for the culture of GSCs, tissues from patient 
specimen were acutely minced after sampling. The tissues were then washed, mechanically dissociated by repetitive pipette. Single cells were primarily plated in serum-free Dulbecco's modified Eagle's medium (DMEDM)/F12 medium containing $20 \mathrm{ng} / \mathrm{ml}$ basic fibroblast growth factor (bFGF, human recombinant, Sigma), $20 \mathrm{ng} / \mathrm{ml}$ epidermal growth factor (EGF, mouse submaxillary), the B-27 (1:50, GIBCO), penicillin (100 $\mathrm{U} / \mathrm{ml})$ and streptomycin $(0.1 \mathrm{mg} / \mathrm{ml})$. Cells were cultured at a density of $1 \times 10^{5} \mathrm{cell} / \mathrm{ml}$ in 24 -well plates $(0.5 \mathrm{ml} /$ well $)$, and were fed every 3 days by adding fresh medium supplemented with GSI or DMSO with indicated concentrations. Animal experiments were reviewed and approved by the Animal Experiment Administration Committee of the Fourth Military Medical University.

\section{Neurosphere assays}

After 7 days from primary culture the numbers of primary spheres were counted under a microscope (Additional file 1: Figure S2) [21]. And for the expression of target genes, neurospheres were harvested on the 5th day of culture for RNA extraction, cDNA synthesis, and real-time reverse transcription-polymerase chain reaction (RT-PCR). Primary neurospheres were harvested and dissociated mechanically into single cell suspensions, and were replated at $1 \times 10^{5}$ cells $/ \mathrm{ml}$ in 24 -well plates. Cells were then cultured for another 7 days until secondary spheres formed [31], which were quantified by counting. On the 7th day of primary culture, neurospheres were plated onto poly-D-lysine (Sigma) coated glass cover slips in DMEM/F12 containing 10\% fetal bovine serum (FBS) for another 7 days. On the third day of differentiation, neurospheres were photomicrographed and their neurites were counted and measured, then on the 7th day of differentiation culture, immunofluorescence staining was performed as described below.

\section{Immunofluorescence}

Undifferentiated neurospheres were plated onto poly-Dlysine coated glass cover slips in serum-free medium for $4 \mathrm{~h}$. Then cells were directly fixed in $4 \%$ paraformaldehyde at $4^{\circ} \mathrm{C}$ for $10 \mathrm{~min}$, and incubated with primary antibodies overnight at $4^{\circ} \mathrm{C}$, followed by species-specific secondary antibodies. Samples were visualized under fluorescence microscope (FV-1000, Olympus, Japan). Immunofluorescence for differentiated neurospheres was performed in a similar way. Cells were additionally counterstained with Hoechst. Primary antibodies used included rabbit anti-Nestin serum (1:200, Sigma), rabbit anti-glial fibrillary acidic protein (GFAP, 1:200, Sigma), mouse anti-mitogen-activated protein 2 (MAP2, 1:200, Sigma). FITC-conjugated goat anti-mouse IgG and Cy3- conjugated goat anti-rabbit IgG (1:400, Jackson ImmunoResearch) were used as the secondary antibodies.

\section{Quantitative RT-PCR}

Total RNA of neurospheres was isolated using the Trizol reagent (Invitrogen). cDNA was synthesized and was used for real-time PCR with a kit (SYBR Premix EX Taq, Takara, Kyoto, Japan) and the ABI PRISM 7300 real-time PCR system, with human GAPDH and mouse $\beta$-actin as the reference controls. Primers used for realtime PCR were summarized in Additional file 1: Table S3.

\section{DNA content analysis}

Spheres were dissociated mechanically into single cell suspensions in the culture medium. Cells were then washed and resuspended in PBS, and were fixed with ethanol at room temperature for $20 \mathrm{~min}$. Cells were resuspended in PBS containing $50 \mu \mathrm{g} / \mathrm{ml}$ of propidium iodide and $0.1 \mathrm{mg} / \mathrm{ml}$ RNase A for $10 \mathrm{~min}$, and were analyzed for ploidy using a flow cytometry (BD Biosciences). Data analysis was performed using the CellQuest software (BD Biosciences).

\section{Statistics}

Independent cultures from at least three samples were used for each experiment (Additional file 1: Table S2). For immunofluorescence, cells were counted by ImageProPlus 6.0, and only cell bodies that were labeled with immunoreactivity were included. Proportions of immunoreactive cells in the total population of cultured cells revealed by Hoechst staining were calculated, and at least 5 microscopic fields per specimen were selected. For neurite analysis, neurites of 30 neurospheres from each culture in the presence of GSI or DMSO were measured. The total numbers of neurites per tumor spheres were counted via photomicrographs taken by a phase contrast microscopy, and the average of the length of neuritis per tumor spheres were measured by Image-ProPlus 6.0. Each experiment was repeated for at least three times. Data were expressed as mean \pm s.e.m, and the difference between the two groups was analyzed with the Student's t-test, with $P<0.05$ as statistically significant.

\section{Results}

\section{Formation of neurosphere-like colonies from primary glioma specimens}

Nine specimens of gliomas were used in the current studies, including 3 oligoastrocytomas, 3 oligodendrogliomas, 2 astrocytomas, and 1 glioblastoma, and the specimens were graded according to the WHO grading scheme (Additional file 1: Table S1). 
Tumor tissues were dissociated mechanically into a single cell suspension and were cultured in serum-free DMEM/F12 medium supplemented with EGF and bFGF. Seven primary gliomas of the nine gave rise to proliferating tumor spheres. Regardless of pathological subtype and grade, neurosphere-like clusters, or tumor spheres, first appeared within $72 \mathrm{~h}$ of primary culture and increased their numbers and diameters quickly during 7 days after the onset of the culture (Figure 1A). In order to estimate whether these tumor spheres showed NSC properties, we stained the tumor spheres from patients with anti-Nestin antibody. The result showed that these tumor spheres expressed Nestin, a marker of NSCs (Figure 1B). The multipotency of these human glioma cell-derived tumor spheres was confirmed by differentiation assay in vitro. We estimated the differentiation capacity of tumor spheres in differentiating conditions by examining the types of molecular markers expressed by neurons and glial cells. We observed that these cells could differentiate into GFAP-positive astrocyte- and MAP2-positive neuron-like cells (Figure 1C, 1D). In addition, a local recurrence tumor also could produce tumor spheres in growth medium (data not shown). Tumor spheres could be passed at least for five generations by mechanical dissociation and their stemness and multipotency could be maintained in serumfree medium supplemented with growth factors for at least one month.

Blockade of Notch signaling attenuates the proliferation and self-renewal ability and promotes differentiation of normal NSCs

Stem cell-like cells in brain tumors share many similarities with normal neural stem/progenitor cells and may
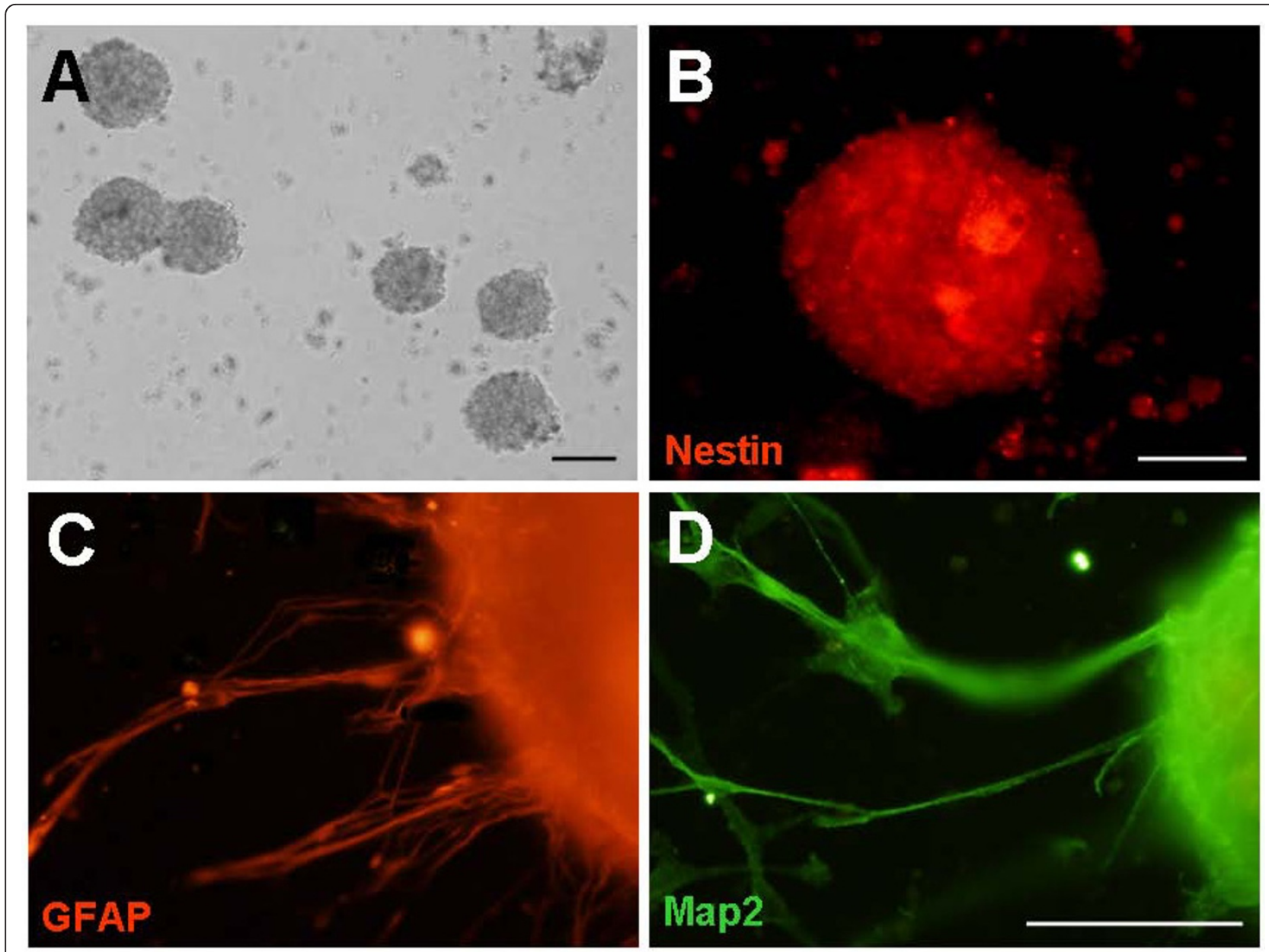

Figure 1 Patient glioma-derived stem cells have the ability to form neurosphere-like colonies and gave rise to the downstream neural cell types of NSCs. (A) Photomicrographs of typical primary tumor spheres from one glioma tissue at $72 \mathrm{~h}$ after plating. (B) Undifferentiated primary tumor spheres expressed high levels of Nestin (red), a marker of NSCs. (C, D) The tumor spheres-derived from human glioma were cultured in differentiation conditional medium for 7 days, and differentiated into neural cells expressing specific molecular markers of GFAP (C, red) and MAP2 (D, green). Scale bar, $100 \mu \mathrm{m}$ in $A$, and $50 \mu \mathrm{m}$ in BCD. 


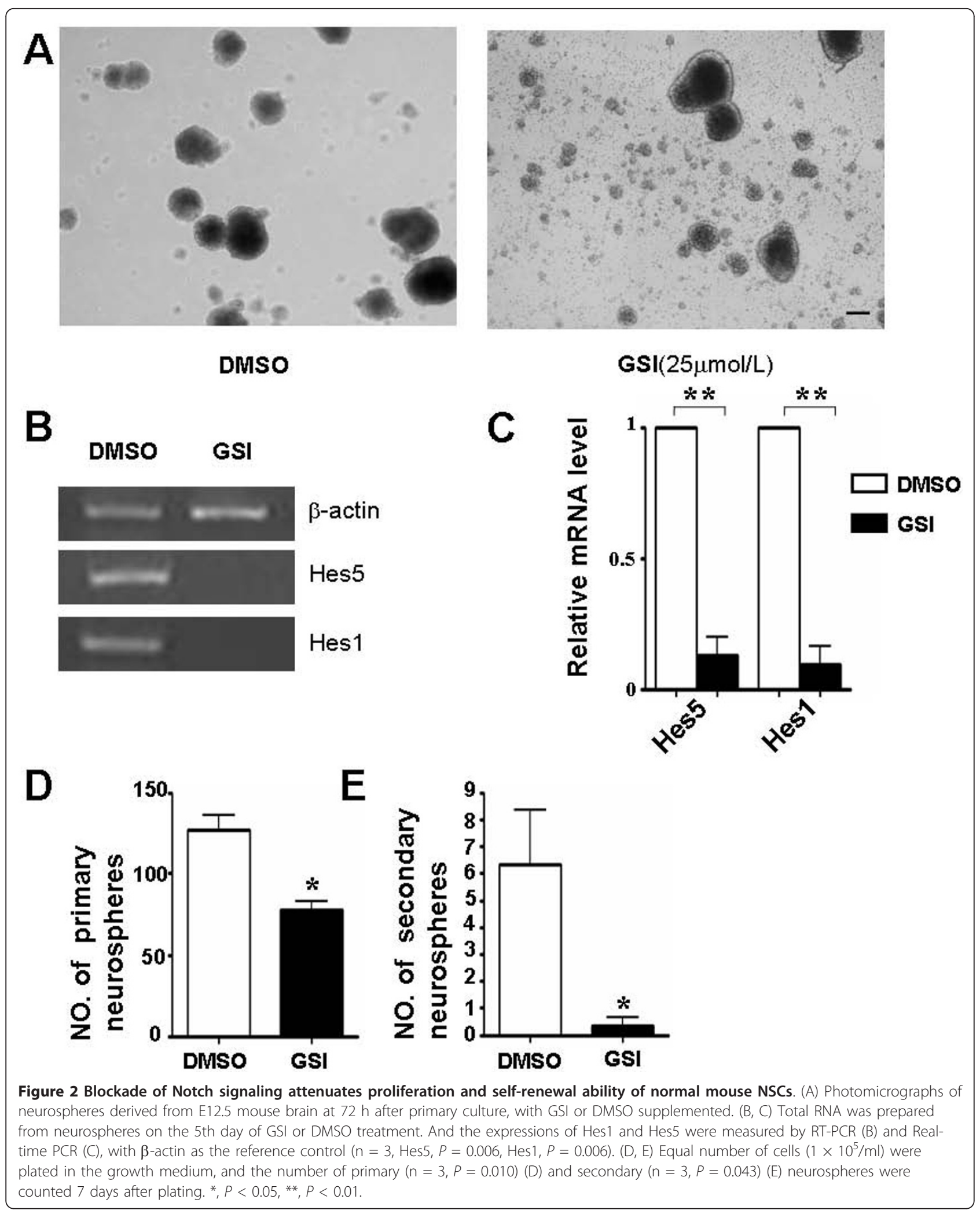


require Notch signal for their survival and growth. In vitro, NSCs proliferate and form clonal spheres referred to as neurospheres. GSI reduced the proliferation of mouse embryonic brain-derived NSCs in a dose-dependent fashion (Additional file 1: Figure S1). The number of neurospheres was decreased in the presence of GSI, compared with the control treated with DMSO (Figure 2A). In order to confirm that GSI effectively blocked Notch signaling in NSCs in our culture system, we test the expression of Hes 1 and Hes5, both of which are downstream molecules of the Notch signaling [12]. Total RNA was prepared from neurospheres on the fifth day of $25 \mu \mathrm{mol} / \mathrm{L}$ GSI treatment and was used for RTPCR. The expression of Hes 1 and Hes 5 decreased remarkably in NSCs, suggesting that GSI at this concentration could inhibit Notch signaling effectively (Figure $2 \mathrm{~B}, 2 \mathrm{C}$ ). We quantitatively analyzed the number of primary neurospheres in the presence of GSI, and found that there was a significant decrease in the number of neurospheres upon GSI treatment at $25 \mu \mathrm{mol} / \mathrm{L}$ (Figure 2D). In order to determine the possible effect of GSI on the NSCs self-renewal ability, we harvested the spheres and dissociated them into a single cell suspension by soft pipeting. When replated in the presence of GSI, the number of secondary neurospheres significantly decreased after 7 days culture (Figure 2E). These results suggested that the proliferation of NSCs was slowed by inhibiting Notch signaling and the self-renewal ability, a key NSC behavior, was at least partially depleted.

Notch signaling has been shown to inhibit the differentiation of NSCs to INPs [21]. In our study, we tested the expression of molecular markers of INPs in primary neurospheres treated with GSI or DMSO. Quantitative RT-PCR showed that the mRNA levels of Glast, which is indicative of the frequency of NSCs, were decreased, while that of Mash 1 and Tubulin $\alpha 1$, both of which are markers of INPs, were increased (Figure 3A, 3B). These results indicated an augmented differentiation from NSCs into INPs upon the blockade of Notch signaling by GSI.

To further study the effect of inhibiting Notch signaling on NSC differentiation, we used the neurosphere differentiation assay in vitro. When spheres were cultured adherently on poly-D-lysine coated glass cover slips without growth factors, they began to differentiate into cells bearing specific markers of neurons and astrocytes. We quantitatively compared the cell types produced by neurospheres in the GSI-containing medium with that of the control. All of the neurospheres gave rise to cells with the molecular markers of neurons or astrocytes (Figure 3C, 3D). However, the percentage of $\mathrm{MAP}^{+}$cells increased significantly in the presence of GSI, from $29.0 \pm 10.4 \%$ to $66.5 \pm 8.4 \%$, and the percentage of $\mathrm{GFAP}^{+}$cells in GSI-treated neurospheres was elevated from $8.7 \pm 3.0 \%$ to $26.9 \pm 6.6 \%$ (Figure 3E, 3F). These results suggested that inhibiting Notch signaling in NSCs leads to an increase in the number of differentiated cells.

\section{Decreased proliferation and self-renewal ability of GSCs upon GSI treatment}

Although Notch signaling has been shown to play critical roles in the maintenance of normal NSCs, whether this signaling might be involved in tumor stem cells is not fully clear. To determine whether Notch signaling activity was required during growth of GSCs, we investigate the effect of GSI on proliferation and self-renewal of GSCs. After Notch signaling was inhibited in GSCs by GSI treatment at $25 \mu \mathrm{mol} / \mathrm{L}$, the expressions of Hes 5 and Hes1, the specific and direct downstream targets of the Notch/RBP-J transcription complex were identified by RT-PCR and real-time PCR as described previously. After 5 days of GSI treatment, Hes 5 and Hes 1 expression markedly decreased (Figure 4A, 4B), and no obvious cell death was observed, indicating no effect on cell viability (data not shown). These results indicated that Notch signaling was efficiently blocked by GSI treatment in GSCs.

Next, we quantitatively compared the proliferation and self-renewal ability of GSI-treated GSCs with that of the controls. The number of the primary tumor spheres in the presence of GSI decreased significantly, from $51.5 \pm$ 2.8 to $34.8 \pm 3.3$ (Figure 4 C). Self-renewal ability of the tumor spheres was assayed by dissociating and replating the primary tumor spheres. Our results showed that GSI-treated GSCs generated a decreased number of secondary tumor spheres $(17.5 \pm 2.3)$, than the number of controls $(31.7 \pm 5.6)$ (Figure 4D). These results showed that the proliferation and self-renewal ability of GSCs also could be attenuated by inhibiting Notch signaling.

\section{Blockade of Notch signaling promotes the differentiation of GSCs}

The previous result indicated that inhibiting Notch signaling promotes the normal NSCs to differentiate into neurons and astrocytes, both of which are the downstream neural cell types of NSCs. Therefore, we investigated whether the GSI treatment promoted GSCs differentiation. Interestingly, after 3 days, approximately $18.7 \pm 0.9$ neurites grew out from each tumor spheres cultured in the medium with GSI, compared to only 6.7 \pm 0.9 from that cultured with DMSO. Meanwhile, the average length of neurites increased from 206.0 \pm 13.1 $\mu \mathrm{m}$ in tumor spheres culture with DMSO to $269.7 \pm$ $28.4 \mu \mathrm{m}$ in GSI-treated tumor spheres (Figure 5B, 5C). In order to further confirm whether these cells are the downstream neural cell types, immunofluorescence was performed on differentiated primary GSCs using the 


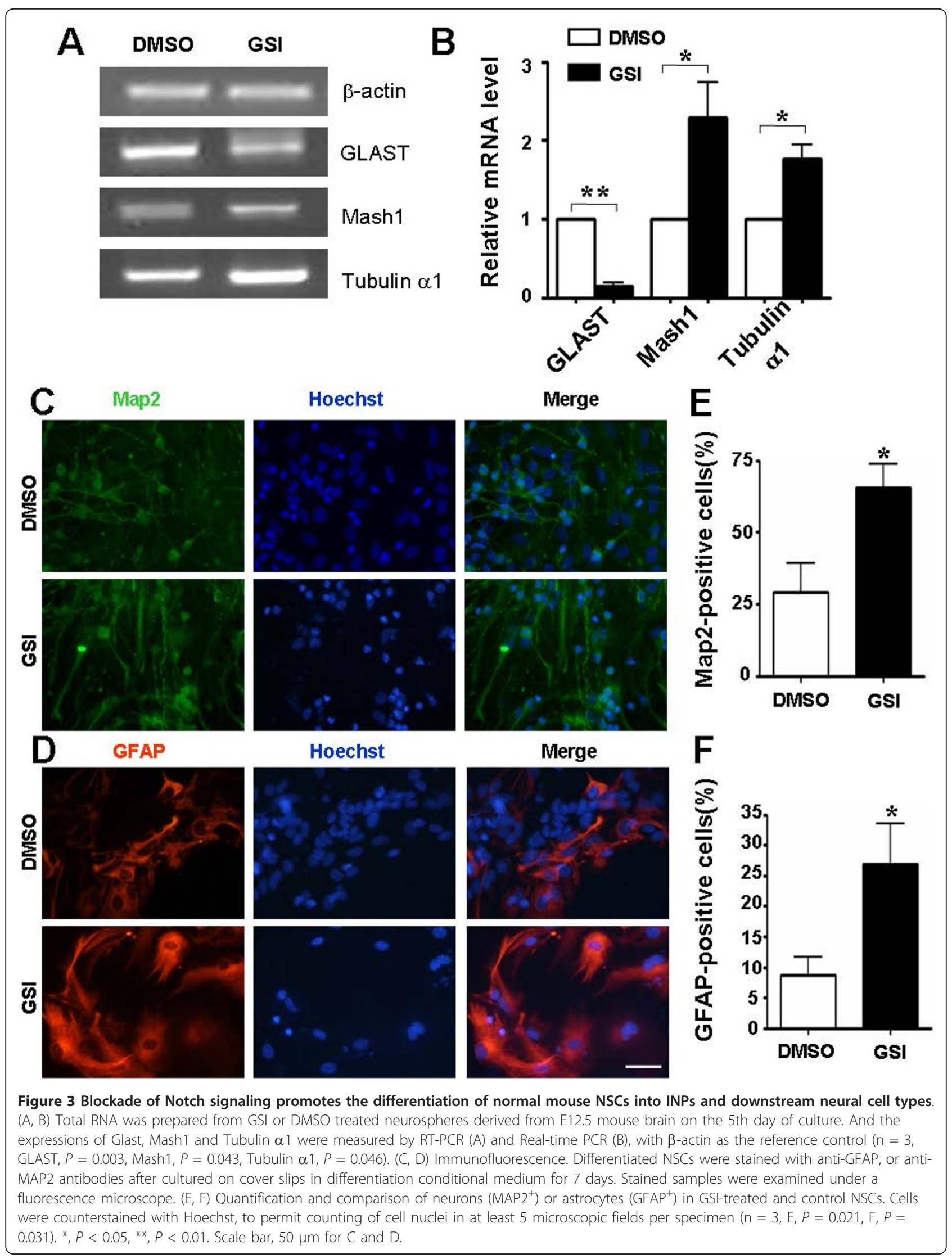




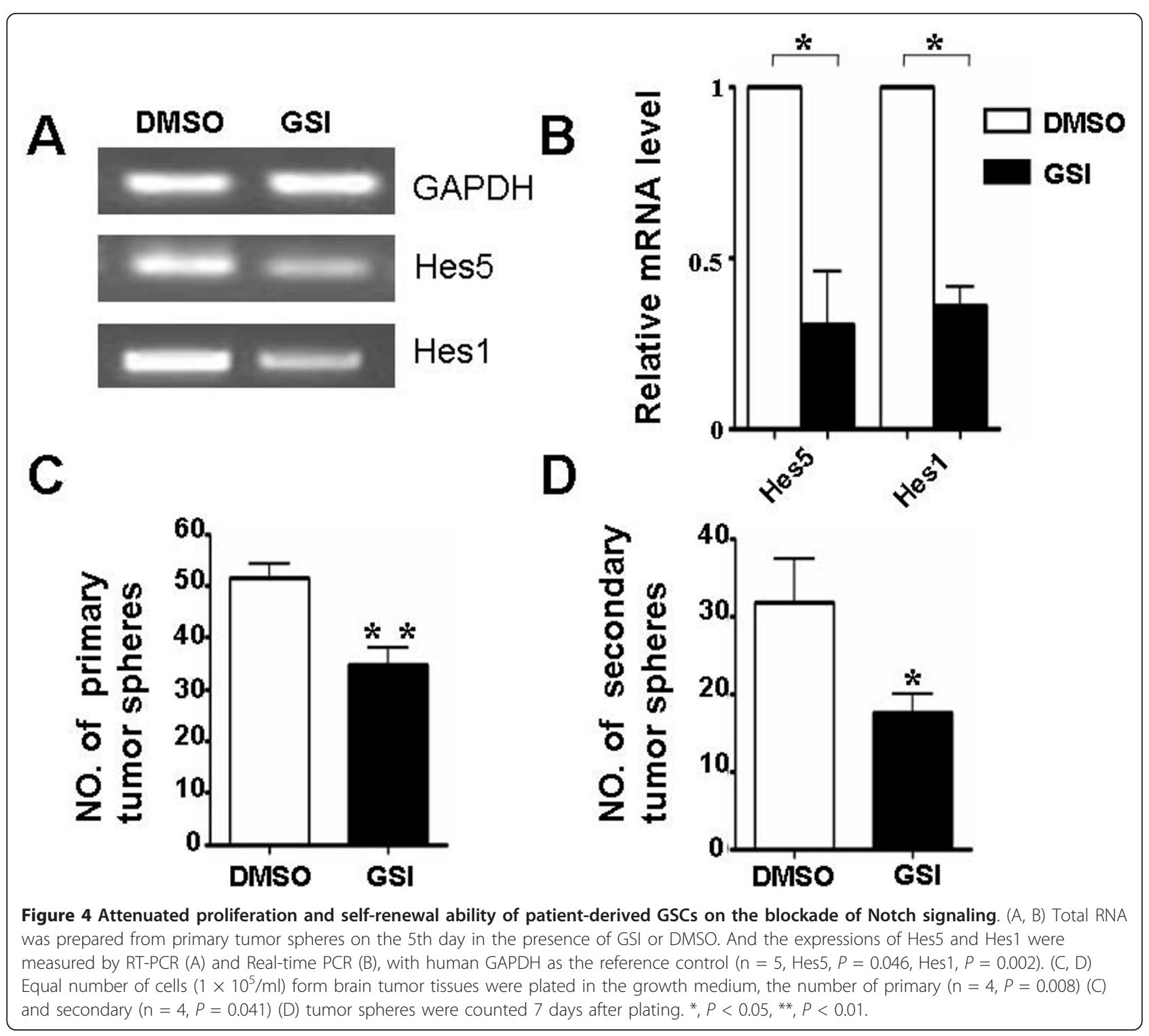

specific markers of neurons and astrocytes on the 7th day in differentiating conditional medium (Figure 5D, $5 \mathrm{E})$. We quantitatively compared the cell types produced by neurospheres in the GSI-treated group with that of the control. The percentages of $\mathrm{MAP}^{+}$cells and $\mathrm{GFAP}^{+}$cells increased significantly, as high as $51.6 \pm$ $6.1 \%$ and $44.0 \pm 1.7 \%$, respectively (Figure 5F, 5G). These results suggest that inhibiting Notch signaling also promotes the differentiation of GSCs.

\section{Blockade of Notch signaling promotes the conversion of} GSCs to INP-like cells

The previous report indicated that blockade of Notch signaling in the CNS increased the frequency of INPs in vivo [21]. Precocious differentiation of NSCs into INPs might exhaust the NSC pool. Therefore, we investigated the effect of inhibiting Notch signaling on the frequency of GSCs and INP-like cells in glioma specimen. In an attempt to distinguish GSCs and INP-like tumor cells, we examined the expression of several markers that could distinguish NSCs from INPs by quantitive RTPCR $[20,21]$. Compared with the controls, the primary tumor spheres in the presence of GSI expressed lower Glast and CD133, which are indicative of the frequency of NSCs and GSCs. In contrast, Mash1 was highly expressed in GSI-treated tumor spheres (Figure 6A, 6B), although the expression level of another INP marker, Tubulin $\alpha 1$ was comparable between the GSI-treated tumor spheres and that of control. Altogether, these results suggested that blockade of Notch signaling may 

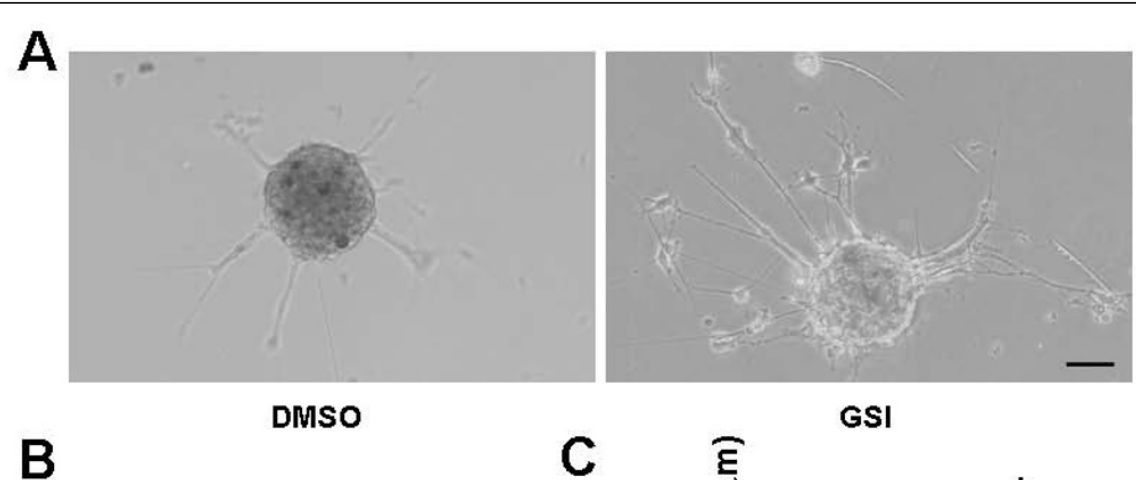

B

C
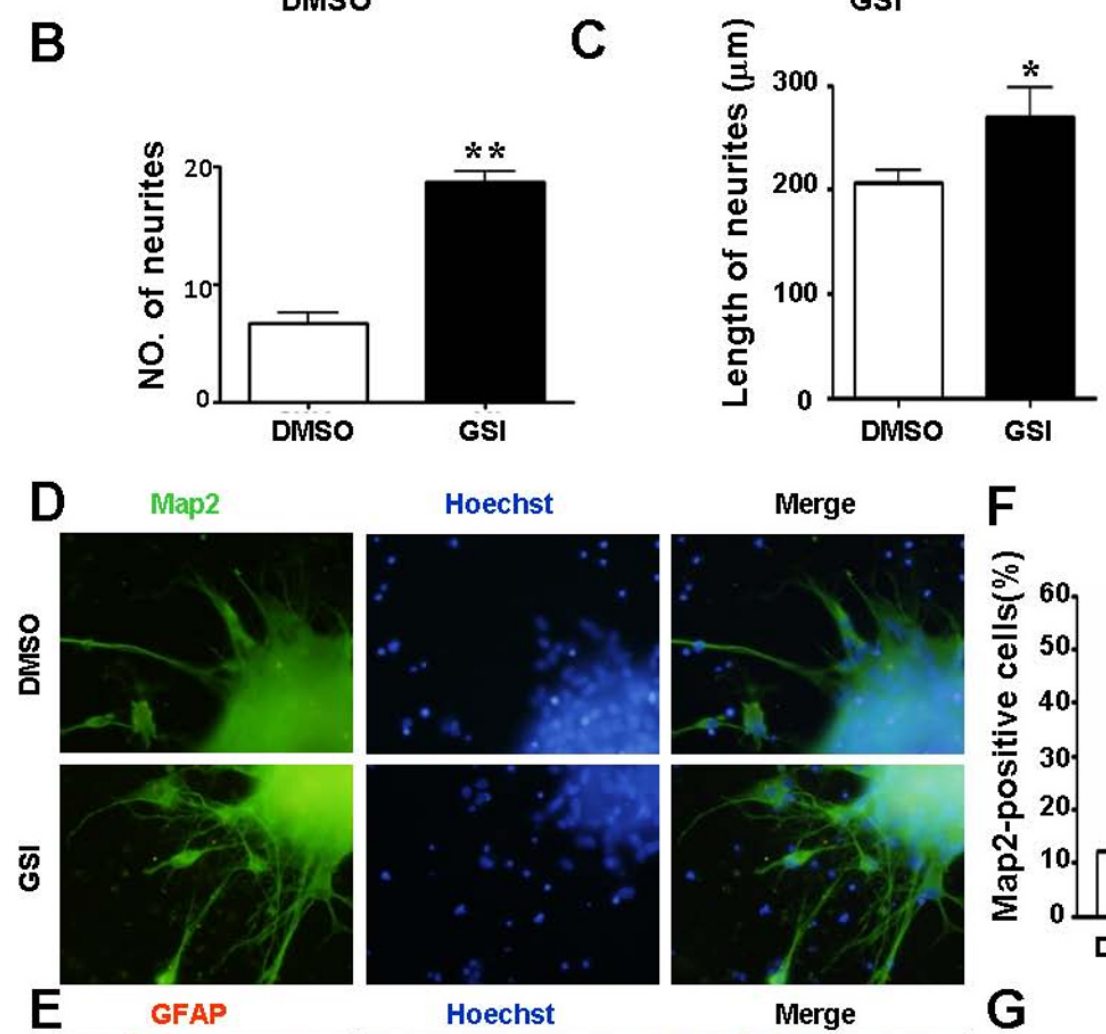

Hoechst
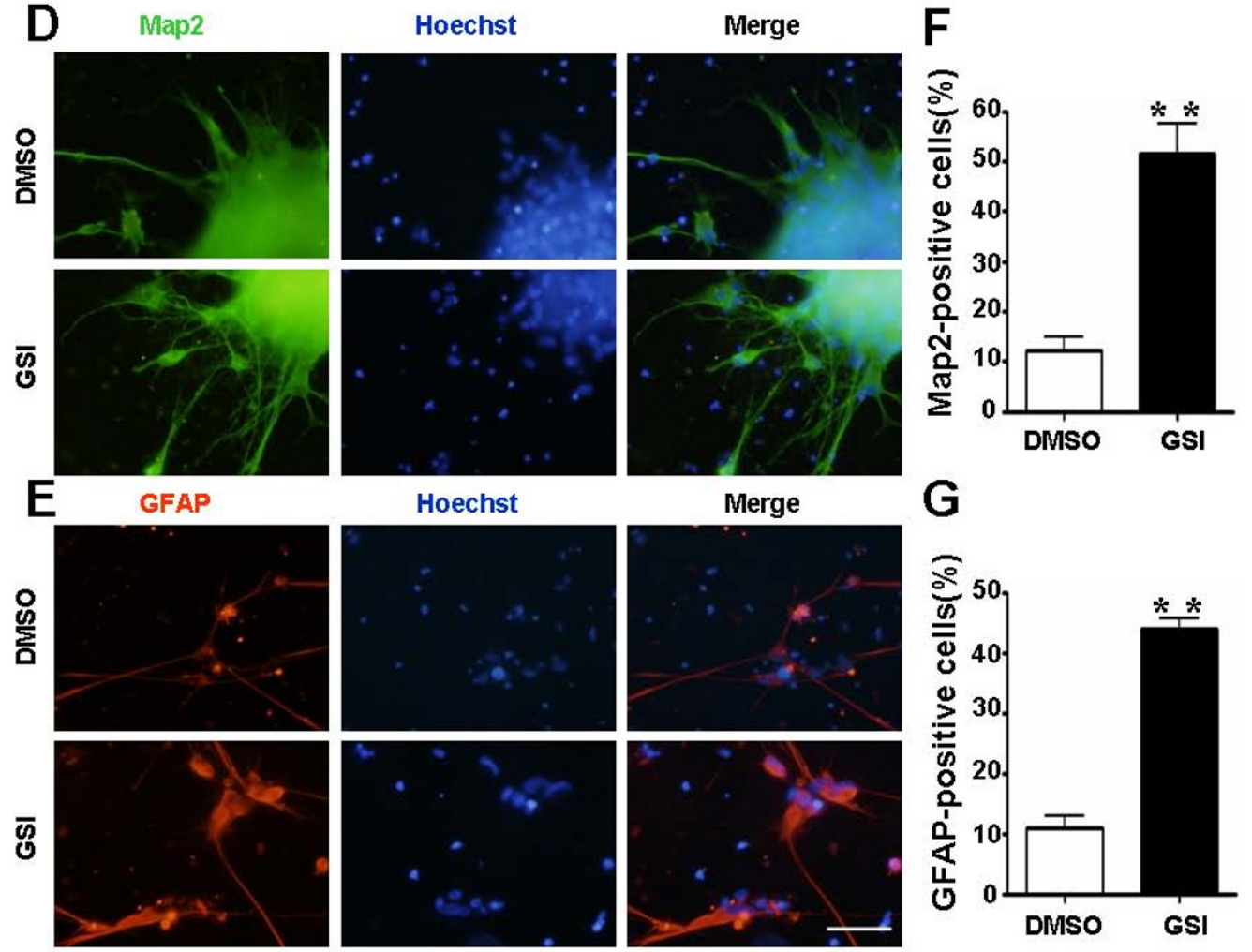

Figure 5 Augmented neurite outgrowth and enhanced differentiation of patient-derived tumor spheres on the blockade of Notch signaling. (A) Photomicrographs of differentiated tumor spheres at $72 \mathrm{~h}$ after plated in differentiation conditional medium supplemented with GSI or DMSO. (B, C) Comparison of neurites number $(n=3, P<0.001)(B)$ and length $(n=3, P=0.041)(C)$ between tumor spheres in the presence of GSI and DMSO. (D, E) Immunofluorescence. Differentiated tumor spheres were stained with anti-GFAP, or anti-MAP2 antibodies after cultured on cover slips in differentiation conditional medium for 7 days. Stained samples were examined under a fluorescence microscope. $(F, G)$ Quantification and comparison of the percentages of neurons (MAP2 $\left.{ }^{+}\right)(n=3, P<0.001)(F)$ or astrocytes $\left(G F A P^{+}\right)(n=3, P<0.001)(G)$ in the total cell number revealed by Hoechst counterstaining, between GSI-treated and control GSCs. Scale bar, $100 \mu \mathrm{m}$ for A, and $50 \mu \mathrm{m}$ for D and E. $*, P<0.05, * *, P<0.01$ 


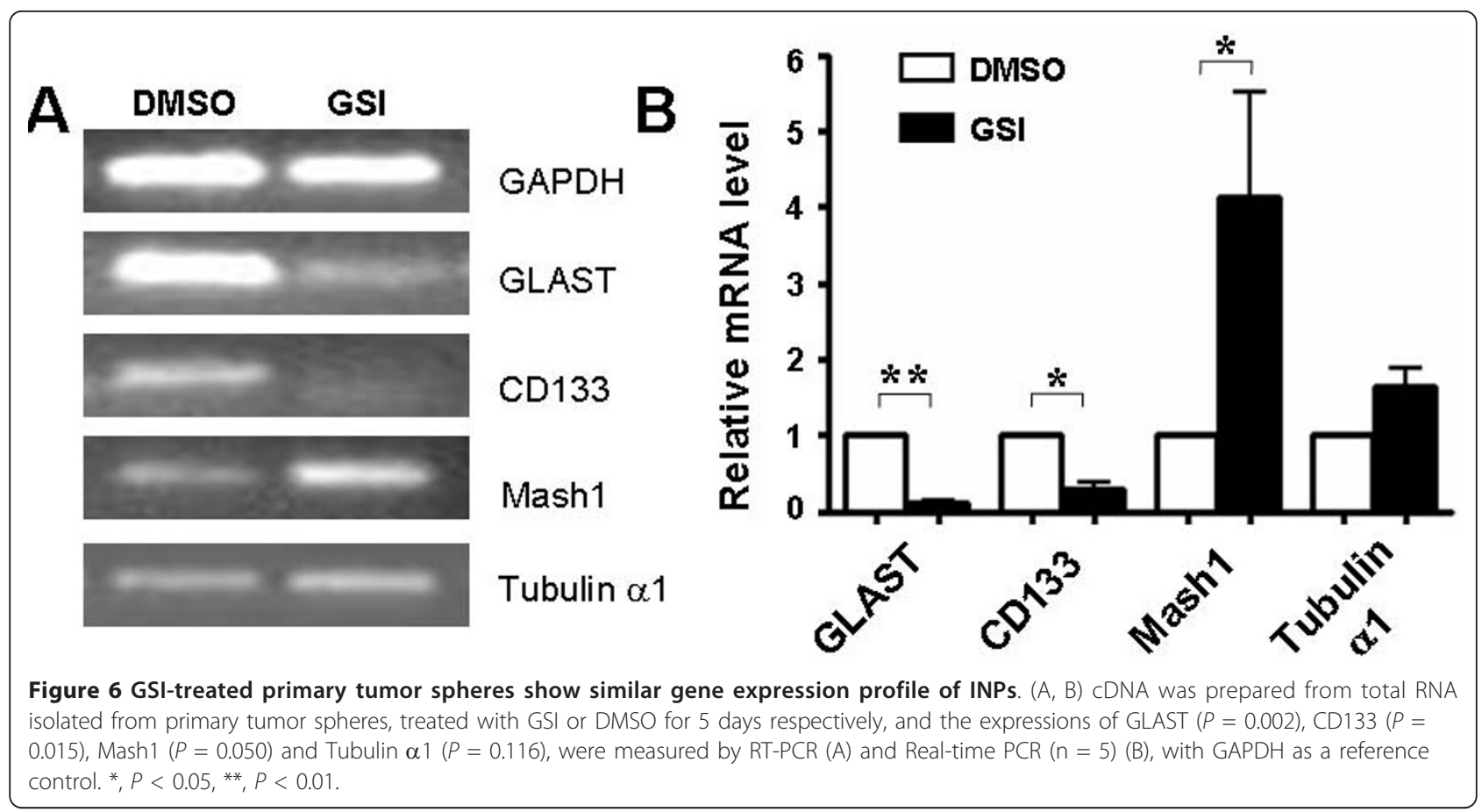

promote the conversion of GSCs to INP-like tumor cells.

\section{GSCs show resistance to GSI treatment compared with NSCs}

To gain further perspective on the dynamics of cellular proliferation accompanying differentiation, we treated NSCs and tumor spheres at a series of time points following GSI treatment with propidium iodide and examined cell cycle via FACS analysis. Compared with the controls, nearly $15.5 \pm 0.5 \%$ of the NSCs treated with GSI for $24 \mathrm{~h}$ are in the G2+M phase, and then sharply decreased to less than $8.2 \pm 1.7 \%$ at $72 \mathrm{~h}$ (Figure $7 \mathrm{~A}$ ). In contrast, the ratio of GSCs in the $\mathrm{G} 2+\mathrm{M}$ phase were slightly elevated at $48 \mathrm{~h}$, and then declined insignificantly at $72 \mathrm{~h}$ (Figure 7B). The result showed that GSI treatment significantly reduced the ratio of the $\mathrm{G} 2+\mathrm{M}$ phase NSCs, but there is no obvious effect on the cell cycle of GSCs. Therefore, NSCs are more sensitive to GSI, while GSCs display a certain degree of resistance to GSI at the early stage of the treatment.

\section{Discussion}

Tumor stem cells such as GSCs have been considered as a novel target for the therapy of the malignant tumors, because these cells are supposed to play an important role in tumor initiation, growth, and recurrence [4]. Similarities in the growth characteristics and gene expression patterns of normal NSCs and GSCs suggest that pathways

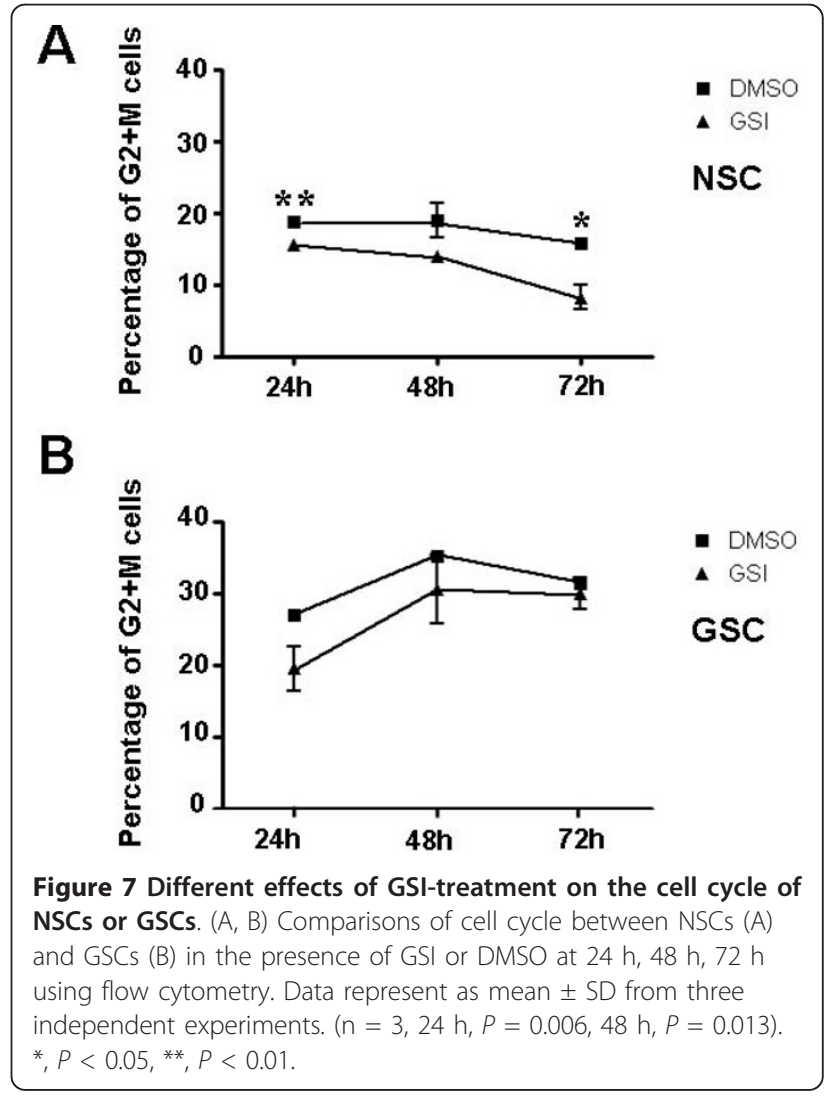


important for NSCs are probable targets for oncogenic brain tumor stem cells. In the present study, 1) we isolated GSCs from the human glioma tissues; 2) Like NSCs, these cells had the ability to form spheres in serum-free medium supplemented with growth factors and differentiated into downstream neural cell-like cells; 3) By GSI treatment, the number of GSCs-derived primary neurospheres and secondary neurospheres were markedly reduced compared with those treated with DMSO, indicating that in the long term culture (7-14 days), the proliferation and self-renewal ability of GSCs was ultimately reduced, upon the blocking of Notch signaling; 4) However, within 72 h culture, GSCs showed a certain degree of GSI-resistance, with undisturbed proliferation ability upon GSI treatment; 5) In addition, we showed that on blocking Notch signaling, GSCs are much biased to differentiate into INP-like cells, and ultimately neurons and glial cells in vitro. All these results suggest a promising preclinical application of Notch signaling antagonist (e.g., GSI)-based CSCs-targeting therapy in malignant glioma patients.

\section{The frequency of GSCs in tumor tissue}

Although CSCs have been identified as an important factor in tumor initiation and growth, their characteristics remain obscure concerning their heterogeneity. Here we found in our experiments that although seven of the nine human gliomas gave rise to proliferating tumor spheres, different numbers of spheres arisen from equal primary glioma cells among tumor samples. It should be noted that the specimens which did not give rise to proliferating neurospheres were patient \#1 (oligoastrocytoma, gradeII) and \#7 (anaplastic astrocytoma, grade III), with comparable tumor grades with the other specimens (Additional file 1: Table S1). Because samples are usually drawn from the periphery of the ablated tumor bulk, these two specimens might contain a certain amount of normal tissues. Overall, equal number of cells from high-grade and recurrent tumors, such as giant cell glioblastoma (WHO grade IV) and oligoastrocytoma (WHO grade III, recurrent tumor) often generate more primary tumor spheres. Due to limited number of samples, our accumulated results could not statistically lead to the conclusion that high-grade tumors contain more GSCs at present. However, the tendency described above indicated that the original frequency of GSCs might be different among samples according to tumor grades, or the GSCs from high-grade tumor tissues might show more typical properties of stem cells with higher proliferation and self-renewal ability.

\section{INP-like cells in GSCs population}

In normal development of the brain, neurons and glia are generated from both NSCs and more limited INPs. And blockade of Notch signaling in NSCs have been shown to promote their conversion into INPs [20,21]. GSCs can differentiate into neurons and astrocytes in culture medium with serum, as shown by our results and previous studies [4]. Like INPs, it is possible that an intermediate glioma progenitor cells (IGPs) also exist, linking the GSCs-IGPs-Neuron/glia hierarchy in tumor microenvironment [32]. Our results show that blocking Notch signaling in the primary tumor spheres leads to down-regulated mRNA level of CD133, a well accepted marker of GSCs at present, indicating a decrease of GSCs. Simultaneously, the mRNA level of Hes 5 and Glast, two markers highly expressed in NSCs were also decreased, while that of Mash1, a marker up-regulated in INPs was increased in primary tumor spheres after being treated with GSI [20]. In addition, Tubulin $\alpha 1$, an INP marker, seems can not distinguish IGPs from GSCs. Since GSI-treated primary tumor spheres could still gave rise to secondary spheres, unless much fewer than that derived from control primary spheres, the CD $133^{\text {low }} /$ GLAST $^{\text {low }} /$ Hes $5^{\text {low }} /$ Mash $1^{\text {high }}$ IGPs might exist, with its number increased and proliferating ability decreased after the blockade of Notch signaling. Therefore, inhibiting Notch signaling might have therapeutic potential for human gliomas by exhausting GSCs and instruct them into less proliferative IGPs and differentiated neural cell types.

\section{Double positive cell types in the derivatives of GSCs}

Although tumor-derived stem cells had many similarities to normal NSCs, it is important to note that differences might exist between them. Sphere differentiation assay on the specimen of $4 \#$ patient demonstrated that GSCs could give rise not only to neurons and glia but also to a few cells that expressed both Map2 and GFAP, the molecular markers of astrocyte and neuron, respectively (Figure S3). Previous studies have reported similar abnormal cells in culture derived from pediatric and adult brain tumors [4,33], indicating that such dual-fate cells might represent a significant fraction of GSCs derived progeny. These Map $2^{+} / \mathrm{GFAP}^{+}$cells sometimes appeared larger than other cells derived from the same sphere (Figure S3). In addition, the GFAP positive glial cells derived from GSCs showed abnormal morphology, with slim cell bodies and neurites, compared with that derived from NSCs (Figure 3D, Figure 5E). Although morphological differences might exist between mouse and human glial cells, previous research on normal human tissue demonstrated that GFAP staining of human glial cells showed similar morphology with that of mouse glial cells [34]. Therefore, the morphological difference of GFAP positive glial cells might be attributed to whether they are NSC-derived or GSC-derived. Genetically, the generation of the double-positive cells and dysmorphic glial cells may accompany with gene 
mutation or abnormal activation of some signal pathways, leading to aberrant reprogramming procedure of GSCs, compared with normal differentiation of NSCs.

GSI-resistance of GSCs at the early stage of GSI treatment In our study, we found that the numbers of both primary and secondary tumor spheres were decreased in the long run (7-day culture) after GSI treatment compared with the controls. However, cell cycle analysis results showed that although Notch blockade significantly reduced the ratio of the $\mathrm{G} 2+\mathrm{M}$ phase in NSCs, there is no obvious effect on the percentage of proliferating GSCs within $72 \mathrm{~h}$ after GSI treatment. These results indicate that, compared with NSCs, another distinctive feature of GSCs was that the former are more sensitive to GSI, while the latter displays a certain degree of resistance to GSI treatment at the early stage of the treatment. Due to the limited amount of primary glioma specimens, the cell cycle analyses were executed on primary tumor spheres from three independent tumor samples. Therefore, the resistance to GSI in GSCs at the early stage of the cell cycle might be a general characteristic in gliomas, or it only represents a few cases of glioma patients which might display resistance in the preclinical trial of GSI treatment. Previous research show that treatment with dipeptide GSI resulted in a marked reduction in medulloblastoma growth [35]. More recently, a clinical trial for a Notch inhibitor, MK0752 (developed by Merck, Whitehouse Station, NJ), has been launched for T-cell acute lymphatic leukemia and breast cancer patients (http://www.clinicaltrials.gov/ct/show/NCT00100152). Although GSI seems to be a promising reagent targeting GSCs by interfering Notch signaling, our results suggested that its effect might be limited to some glioma patients. Therefore, drug combination should be used at the early stage of therapy. However, since our results are based on in vitro culture system of patient-derived samples, more accurate conclusion could be drawn from animal models or preclinical trials in future study.

\section{The mechanisms of Notch signaling in regulating the proliferation and differentiation of GSCs}

The mechanistic links between Notch signaling and the proliferation and differentiation of GSCs were presumably governed by more than one mechanism. In our study, the decreased proliferation and increased differentiation of GSCs upon GSI treatment are accompanied with downregulation of Hes1 and Hes5, the canonical Notch downstream effectors. In addition, the expression level of Mash1, a proneural gene antagonized by the Hes genes was up-regulated in GSI-treated primary tumor spheres. Therefore, the canonical Notch-CBF1-Hes axis seems also play critical roles in the proliferation and differentiation of GSCs, as its function in NSCs [11].

On the other hand, Notch signaling has been shown to have both negative and positive influences on cell cycle progression $[11,36]$. In the present study, we observed that the proliferation of GSCs decreased significantly in the long term culture, although GSI resistance of three glioma samples was present (see above). Mutations of p53, pTEN and H-Ras, have been identified in tumor tissues of giloma patients. And Notch signaling has been shown to crosstalk with p53 and pTEN signaling pathway, two major regulators of cell cycle $[37,38]$. In addition, down-regulation of Notch signaling in $\mathrm{H}$ Ras-transformed human breast cells led to a significant decrease in their proliferation [39]. Therefore, how Notch signaling promotes the cell cycle of GSCs is yet to be explored, on the scenery of the complex signal crosstalk and genetic circuitry.

\section{Conclusion}

Our data indicate that like NSCs, Notch signaling maintains the patient-derived GSCs by promoting their self-renewal and inhibiting their differentiation, and support that Notch signal inhibitor might be a prosperous candidate of the drug treatment targeting CSCs for gliomas, however, with GSI-resistance at the early stage of treatment.

\section{Additional material}

Additional file 1: Hu et al Supplementary materials The file contains

Table S1-S3, Figure S1-S3 and their figure legends

\section{Acknowledgements}

This work was supported by grants from the National Natural Science Foundation of China (30700693, 30973370, 31000485, 81071874) and the Ministry of Science and Technology of China (2009CB521706).

\section{Author details}

${ }^{1}$ State Key Laboratory of Cancer Biology, Department of Medical Genetics and Developmental Biology, Fourth Military Medical University, Chang-Le Xi Street \#17, Xi'an 710032, PR China. ${ }^{2}$ Department of Neurosurgery, Xijing Hospital, Fourth Military Medical University, Chang-Le Xi Street \#17, Xi'an 710032, PR China.

\section{Authors' contributions}

YYH and $\mathrm{MHZ}$ carried out tissue culture, animal experiments and gene expression analyses, participated in study design and manuscript preparation. GC and LLi carried out specimens collection. LLiang, FG and YNW helped histological examination and immunohistochemistry staining. $\mathrm{LAF}$ and $\mathrm{HH}$ designed the study and prepared the manuscript. All authors read and approved the final manuscript.

\section{Competing interests}

The authors declare that they have no competing interests.

Received: 22 October 2010 Accepted: 22 February 2011 Published: 22 February 2011 


\section{References}

1. Bonnet D, Dick JE: Human acute myeloid leukemia is organized as a hierarchy that originates from a primitive hematopoietic cell. Nat Med 1997, 3(7):730-737

2. Al-Hajj M, Wicha MS, Benito-Hernandez A, Morrison SJ, Clarke MF: Prospective identification of tumorigenic breast cancer cells. Proc Natl Acad Sci USA 2003, 100(7):3983-3988.

3. Ma S, Chan KW, Hu L, Lee TK, Wo JY, Ng IO, Zheng BJ, Guan XY: Identification and characterization of tumorigenic liver cancer stem/ progenitor cells. Gastroenterology 2007, 132(7):2542-2556.

4. Hemmati HD, Nakano I, Lazareff JA, Masterman-Smith M, Geschwind DH, Bronner-Fraser M, Kornblum HI: Cancerous stem cells can arise from pediatric brain tumors. Proc Natl Acad Sci USA 2003, 100(25):15178-15183.

5. Singh SK, Clarke ID, Terasaki M, Bonn VE, Hawkins C, Squire J, Dirks PB: Identification of a cancer stem cell in human brain tumors. Cancer Res 2003, 63(18):5821-5828

6. Galli R, Binda E, Orfanelli U, Cipelletti B, Gritti A, De Vitis S, Fiocco R, Foroni C, Dimeco F, Vescovi A: Isolation and characterization of tumorigenic, stem-like neural precursors from human glioblastoma. Cancer Res 2004, 64(19):7011-7021.

7. Yuan X, Curtin J, Xiong Y, Liu G, Waschsmann-Hogiu S, Farkas DL, Black KL, Yu JS: Isolation of cancer stem cells from adult glioblastoma multiforme. Oncogene 2004, 23(58):9392-9400.

8. Wu A, Oh S, Wiesner SM, Ericson K, Chen L, Hall WA, Champoux PE, Low WC, Ohlfest JR: Persistence of CD133+ cells in human and mouse glioma cell lines: detailed characterization of GL261 glioma cells with cancer stem cell-like properties. Stem Cells Dev 2008, 17(1):173-184.

9. Singh SK, Hawkins C, Clarke ID, Squire JA, Bayani J, Hide T, Henkelman RM Cusimano MD, Dirks PB: Identification of human brain tumour initiating cells. Nature 2004, 432(7015):396-401.

10. Reya T, Morrison SJ, Clarke MF, Weissman IL: Stem cells, cancer, and cancer stem cells. Nature 2001, 414(6859):105-111.

11. Louvi A, Artavanis-Tsakonas S: Notch signalling in vertebrate neural development. Nat Rev Neurosci 2006, 7(2):93-102.

12. Fan X, Matsui W, Khaki L, Stearns D, Chun J, Li YM, Eberhart CG: Notch pathway inhibition depletes stem-like cells and blocks engraftment in embryonal brain tumors. Cancer Res 2006, 66(15):7445-7452.

13. Struhl G, Adachi A: Nuclear access and action of notch in vivo. Cell 1998, 93(4):649-660

14. Schroeter EH, Kisslinger JA, Kopan R: Notch-1 signalling requires ligandinduced proteolytic release of intracellular domain. Nature 1998, 393(6683):382-386

15. Jarriault S, Brou C, Logeat F, Schroeter EH, Kopan R, Israel A: Signalling downstream of activated mammalian Notch. Nature 1995 377(6547):355-358.

16. Nye JS, Kopan R, Axel R: An activated Notch suppresses neurogenesis and myogenesis but not gliogenesis in mammalian cells. Development 1994, 120(9):2421-2430

17. Campos LS, Decker L, Taylor V, Skarnes W: Notch, epidermal growth factor receptor, and beta1-integrin pathways are coordinated in neural stem cells. J Biol Chem 2006, 281(8):5300-5309.

18. Hoeck JD, Jandke A, Blake SM, Nye E, Spencer-Dene B, Brandner S, Behrens A: Fbw7 controls neural stem cell differentiation and progenitor apoptosis via Notch and c-Jun. Nat Neurosci 2010, 13(11):1365-1372.

19. Alexson TO, Hitoshi S, Coles BL, Bernstein A, van der Kooy D: Notch signaling is required to maintain all neural stem cell populationsirrespective of spatial or temporal niche. Dev Neurosci 2006, 28(1-2):34-48.

20. Mizutani K, Yoon K, Dang L, Tokunaga A, Gaiano N: Differential Notch signalling distinguishes neural stem cells from intermediate progenitors. Nature 2007, 449(7160):351-355.

21. Gao F, Zhang Q, Zheng MH, Liu HL, Hu YY, Zhang P, Zhang ZP, Qin HY, Feng $L$, Wang $L$, et al: Transcription factor RBP-J-mediated signaling represses the differentiation of neural stem cells into intermediate neural progenitors. Mol Cell Neurosci 2009, 40(4):442-450

22. Tanigaki K, Nogaki F, Takahashi J, Tashiro K, Kurooka H, Honjo T: Notch1 and Notch3 instructively restrict bFGF-responsive multipotent neural progenitor cells to an astroglial fate. Neuron 2001, 29(1):45-55.

23. Aguirre A, Rubio ME, Gallo V: Notch and EGFR pathway interaction regulates neural stem cell number and self-renewal. Nature 2010, 467(7313):323-327.
24. Jang MS, Zlobin A, Kast WM, Miele L: Notch signaling as a target in multimodality cancer therapy. Curr Opin Mol Ther 2000, 2(1):55-65.

25. Fan X, Mikolaenko I, Elhassan I, Ni X, Wang Y, Ball D, Brat DJ, Perry A, Eberhart CG: Notch1 and notch2 have opposite effects on embryonal brain tumor growth. Cancer Res 2004, 64(21):7787-7793.

26. Purow BW, Haque RM, Noel MW, Su Q, Burdick MJ, Lee J, Sundaresan T, Pastorino S, Park JK, Mikolaenko I, et al: Expression of Notch-1 and its ligands, Delta-like-1 and Jagged-1, is critical for glioma cell survival and proliferation. Cancer Res 2005, 65(6):2353-2363.

27. Hulleman E, Quarto M, Vernell R, Masserdotti G, Colli E, Kros JM, Levi D, Gaetani $P$, Tunici $P$, Finocchiaro $G$, et al: A role for the transcription factor HEY1 in glioblastoma. J Cell Mol Med 2009, 13(1):136-146.

28. Zhang XP, Zheng G, Zou L, Liu HL, Hou LH, Zhou P, Yin DD, Zheng QJ, Liang L, Zhang SZ, et al: Notch activation promotes cell proliferation and the formation of neural stem cell-like colonies in human glioma cells. Mol Cell Biochem 2008, 307(1-2):101-108.

29. Hovinga KE, Shimizu F, Wang R, Panagiotakos G, Van Der Heijden M, Moayedpardazi H, Correia AS, Soulet D, Major T, Menon J, et al: Inhibition of Notch Signaling in Glioblastoma Targets Cancer Stem Cells via an Endothelial Cell Intermediate. Stem Cells 2010, 28(6):1019-1029.

30. Shih $\mathrm{AH}, \mathrm{Holland} \mathrm{EC}$ : Notch signaling enhances nestin expression in gliomas. Neoplasia 2006, 8(12):1072-1082.

31. Hitoshi S, Alexson T, Tropepe V, Donoviel D, Elia AJ, Nye JS, Conlon RA, Mak TW, Bernstein A, van der Kooy D: Notch pathway molecules are essential for the maintenance, but not the generation, of mammalian neural stem cells. Genes Dev 2002, 16(7):846-858.

32. Chen R, Nishimura MC, Bumbaca SM, Kharbanda S, Forrest WF, Kasman IM, Greve JM, Soriano RH, Gilmour LL, Rivers CS, et al: A hierarchy of selfrenewing tumor-initiating cell types in glioblastoma. Cancer Cell 2010, 17(4):362-375

33. Ignatova TN, Kukekov VG, Laywell ED, Suslov ON, Vrionis FD, Steindler DA Human cortical glial tumors contain neural stem-like cells expressing astroglial and neuronal markers in vitro. Glia 2002, 39(3):193-206.

34. Svendsen $C N$, ter BM, Armstrong RJ, Rosser AE, Chandran S, Ostenfeld $T$, Caldwell MA: A new method for the rapid and long term growth of human neural precursor cells. J Neurosci Methods 1998, 85(2):141-152.

35. Hallahan AR, Pritchard JI, Hansen S, Benson M, Stoeck J, Hatton BA, Russell TL, Ellenbogen RG, Bernstein ID, Beachy PA, et al: The SmoA1 mouse model reveals that notch signaling is critical for the growth and survival of sonic hedgehog-induced medulloblastomas. Cancer Res 2004, 64:7794-7800.

36. Go MJ, Eastman DS, Artavanis-Tsakonas S: Cell proliferation control by Notch signaling in Drosophila development. Development 1998 125(11):2031-2040

37. Dotto GP: Crosstalk of Notch with p53 and p63 in cancer growth control. Nat Rev Cancer 2009, 9(8):587-595.

38. Palomero T, Sulis ML, Cortina M, Real PJ, Barnes K, Ciofani M, Caparros E, Buteau J, Brown K, Perkins SL, et al: Mutational loss of PTEN induces resistance to NOTCH1 inhibition in T-cell leukemia. Nat Med 2007, 13(10):1203-1210.

39. Weijzen S, Rizzo P, Braid M, Vaishnav R, Jonkheer SM, Zlobin A, Osborne BA, Gottipati S, Aster JC, Hahn WC, et al: Activation of Notch-1 signaling maintains the neoplastic phenotype in human Ras-transformed cells. Nat Med 2002, 8(9):979-986.

\section{Pre-publication history}

The pre-publication history for this paper can be accessed here: http://www.biomedcentral.com/1471-2407/11/82/prepub

doi:10.1186/1471-2407-11-82

Cite this article as: Hu et al:: Notch signaling contributes to the maintenance of both normal neural stem cells and patient-derived glioma stem cells. BMC Cancer 2011 11:82. 